\title{
Avaliação de Tecnologias para o Desenvolvimento de Interface RESTful no contexto de Linux Embarcado
}

\author{
${ }^{1}$ Alef Martins, ${ }^{1}$ Marcello Thiry, ${ }^{1}$ Anita Maria da Rocha Fernandes, \\ ${ }^{2}$ Rodrigo de Souza Oliveira \\ ${ }^{1}$ Curso Ciência da Computação - Escola do Mar, Ciência e Tecnologia \\ Universidade do Vale do Itajaí (UNIVALI) \\ Caixa Postal 360 - 88.302-901 - São José - SC - Brasil \\ ${ }^{2}$ MBA em Big Data - FATEC - SENAI - Cuiabá - MT \\ \{alleffyh, rddsouzaoliveira\}@gmail.com, \{thiry, anita.fernandes\}@univali.br
}

\begin{abstract}
Currently many network assets developed with Embedded Linux use RESTful interface as a mean of communication and access to available resources. A critical point in the development of this system model is the scarcity of hardware resources, so great care is needed in the development of applications regarding memory, CPU and disk space usage. Knowing this, this paper aims to make a comparative study of the development models available to assist the reader in deciding which implementation model he should use in the development of this type of system.
\end{abstract}

Resumo. Atualmente vários ativos de rede desenvolvidos com Linux Embarcado utilizam interface RESTful como meio de comunicação e acesso a recursos disponíveis. Um ponto critico no desenvolvimento deste modelo de sistema é a escassez de recursos de hardware, sendo assim é necessário um grande cuidado no desenvolvimento das aplicações quanto ao uso de memória, CPU e espaço em disco. Sabendo disso, este trabalho tem como objetivo realizar um estudo comparativo entre os modelos de desenvolvimento disponíveis para auxiliar o leitor na tomada de decisão sobre qual modelo de implementação ele deve utilizar no desenvolvimento deste tipo de sistema.

\section{Introdução}

Segundo Devine et al. (2019), os últimos anos tiveram um crescimento expansivo do uso de sistemas embarcados. Este crescimento pode ser atribuído principalmente ao surgimento de novas tecnologias que variam como os wearables (como por exemplo os relógios inteligentes), dispositivos para automação residencial e industrial, dispositivos de redes e ao fenômeno conhecido como Internet das Coisas (IoT). Todo esse crescimento e aparição de novos dispositivos demandam um meio de comunicação e troca de informações.

Tendo esta demanda, uma das principais API's (Access Programming Interfaces) em sistemas embarcados é o RESTful (Representation State Transfer). Padmanaban et al. (2018) afirmam que RESTful teve um crescimento muito rápido e se tornou a interface padrão para o desenvolvimento de vários serviços web como por exemplo, interface de comunicação com os sistemas embarcados. Este tipo de interface pode ser implementado de diversas maneiras, com uma variação de linguagens de programação e bibliotecas. 
Segundo Garriga et al. (2015), RESTful é um serviço para criação de interfaces de comunicações entre aplicações utilizando a web. Utilizado para troca de informações entre aplicações no mundo da computação. O serviço utiliza os métodos básicos de requisições HTTP (Hypertext Transfer Protocol), para manipular a troca de informações entre as aplicações.

Porém, o uso de sistemas embarcados exige preocupação quanto a implementação das aplicações e interfaces, pois os recursos de hardware como memória, poder de processamento e espaço de armazenamento geralmente são bem escassos. Assim uma das dificuldades é entender qual modelo de implementação se encaixa melhor com o perfil do hardware em que a interface será implementada. Durante o estudo de trabalhos correlatos não foi encontrado nenhum trabalho que trate com essa problemática de forma direta.

A seguir são apresentados os trabalhos correlatos a esta pesquisa, bem como a proposta, metodologia utilizada e os resultados esperados.

\section{Trabalhos Correlatos}

Durante o desenvolvimento deste trabalho, foram encontrados quatro trabalhos correlatos, que de alguma forma possam contribuir com a elaboração deste estudo. Para a seleção de trabalhos correlatos, foi realizada uma busca informal na literatura com base nos seguintes critérios: (i) Metodologias de avaliações de software; (ii) Sistemas Embarcados; (iii) Testes em aplicações em sistemas embarcados; (iv) Interface RESTful;

Tabela 1. Trabalhos correlatos e suas características

\begin{tabular}{|c|c|c|c|c|c|}
\hline Característica & $\begin{array}{c}\text { FANTIN, Kátia } \\
\text { (2019) }\end{array}$ & $\begin{array}{c}\text { HAMAD e } \\
\text { PREVELAKIS } \\
\text { (2016) }\end{array}$ & $\begin{array}{c}\text { Cheng, } \\
\text { Cheng e } \\
\text { Chen } \\
(2014) \\
\end{array}$ & $\begin{array}{l}\text { Albertengo } \\
\text { et al (2019) }\end{array}$ & $\begin{array}{l}\text { Trabalho } \\
\text { Proposto }\end{array}$ \\
\hline $\begin{array}{l}\text { Metodologia de } \\
\text { Avaliação de } \\
\text { Software }\end{array}$ & Sim & $\mathrm{Sim}$ & Sim & Sim & Sim \\
\hline $\begin{array}{c}\text { Sistemas } \\
\text { Embarcados }\end{array}$ & Não & Sim & Não & Não & $\mathrm{Sim}$ \\
\hline WebService & Não & Não & Sim & Sim & $\mathrm{Sim}$ \\
\hline $\begin{array}{c}\text { Testes de } \\
\text { performance }\end{array}$ & Não & Sim & Sim & Sim & Sim \\
\hline $\begin{array}{l}\text { Critérios de } \\
\text { Comparação }\end{array}$ & Não & Sim & Sim & $\mathrm{Sim}$ & $\mathrm{Sim}$ \\
\hline
\end{tabular}

Todos os trabalhos relacionados tratam de temas bem específicos, e os cinco trabalhos tem como ponto em comum a definição de critérios importantes na avaliação desses temas. Outro fato importante é a comparação entre RESTful e SOAP (outro modelo de API) feita por Cheng et al. (2017), onde ficou explícito o desempenho do modelo RESTful se sobressaindo em relação ao SOAP em um sistema embarcado.

\section{Proposta}

Para solução do problema apresentado no capítulo de introdução, este trabalho tem como objetivo fornecer um estudo comparativo entre principais modelos de implementações de interfaces RESTful no ambiente de Linux Embarcado. Este estudo contemplará uma análise das implementações levando em consideração pilares importantes para o desenvolvimento de uma aplicação voltada para o Linux Embarcado, como por exemplo o uso de memória, tamanho em disco e uso de CPU (Central Processing Unit). 
Estas informações serão coletadas através de uma bancada de testes com diferentes tipos de testes que possam avaliar: (i) Uso de memória em idle e durante testes de requisições; (ii) Uso de CPU durante teste de requisições; (iii) Tamanho em flash da aplicação junto com as bibliotecas necessárias para seu uso;

\section{Metodologia}

Para o desenvolvimento deste estudo, além de uma revisão da literatura serão analisadas as principais bibliotecas de software para o desenvolvimento de interfaces RESTful, o critério básico de seleção é que possa ser empregada no contexto de sistemas embarcados. Na sequência, cada modelo de implementação encontrado será aplicado a uma bancada de testes para que as informações descritas na sessão 3 sejam analisadas. Estas informações serão utilizadas em um método matemático estatístico não paramétrico para auxiliar na definição de qual modelo se destaca em relação a cada um dos parâmetros listados anteriormente.

\section{Resultados Esperados}

O resultado deste estudo poderá ser consumido pelo meio acadêmico e industria e espera-se que o estudo permita ao leitor entender as diferenças entre cada modelo de implementação utilizando ambientes embarcados. E entender os pontos positivos e negativos de cada modelo e todas suas características relacionadas ao desenvolvimento de sistemas embarcados.

Como resultado parcial, o primeiro modelo de implementação utilizado é o framework web ulfius e a linguagem de programação $\mathrm{C}$, onde foi possível alcançar uma média de tempo de resposta em requisições simples de 5,48ms em um ambiente embarcado com uma CPU de 1000Mhz e 64MB de RAM.

\section{Referências}

Albertengo, G., et al. . 2019. On the performance of Web Services, Google Cloud Messaging and Firebase Cloud Messaging. Digital Communications and Networks.

Cheng, Bo, Cheng, Xin, \& Chen, Junliang. 2017. Lightweight monitoring and control system for coal mine safety using REST style. Isa Transaction, 07, 229-239.

Devine, J., Finney, J., de Halleux, P., Moskal, M., Ball, T., \& Hodges, S. 2019. MakeCode and CODAL: Intuitive and efficient embedded systems programming for education. Journal of Systems Architecture, 468-483.

Fantin, Kátia. 2017. Metodologia de Avaliação de Software Educacional. Monografia (Bacharel em Informática), UCS (Universidade de Caxias do Sul), Caxias do Sul, Brazil.

Garriga, M., et al. . 2015. RESTful service composition at a glance: A survey. Journal of Network and Computer Applications, 60, 32-53.

Hamad, M., \& Prevelakis, V. 2015. Implementation and performance evaluation of embedded IPsec in microkernel OS. Pages 1-7 of: WORLD SYMPOSIUM ON COMPUTER NETWORKS AND INFORMATION SECURITY (WSCNIS) 2015. Hammamet.

Padmanaban, R., et al. . 2018. Computability Evaluation of RESTful API using Primitive Recursive Function. Journal Of King Saud University - Computer And Information Sciences, 1-11. 